\title{
A Novel FOXL2 Mutation Implying Blepharophimosis-Ptosis-Epicanthus Inversus Syndrome Type I
}

\author{
Fang Lia,b Peiwei Chaia Jiayan Fan ${ }^{a, b}$ Xi Wang ${ }^{a, b}$ Wenjuan Lu ${ }^{a, b} \quad$ Jin Lia, \\ Shengfang Ge $e^{a, b}$ Renbing Jia ${ }^{a, b}$ He Zhanga ${ }^{a, b}$ Xianqun Fan ${ }^{a, b}$ \\ aDepartment of Ophthalmology, Ninth People's Hospital, Shanghai JiaoTong University School of \\ Medicine, Shanghai, 'Shanghai Key Laboratory of Orbital Diseases and Ocular Oncology, Shanghai, \\ People's Republic of China
}

\section{Key Words}

Bpes $\cdot$ FOXL2 • Premature ovarian failure

\begin{abstract}
:
Background/Aims: Blepharophimosis-ptosis-epicanthus inversus syndrome (BPES) is a rare autosomal dominant disease caused by FOXL2 gene mutations, and it is clinically characterized by an eyelid malformation associated (type I) or not (type II) with premature ovarian failure (POF). Functional study of novel mutations is especially critical for female patients, as it may allow the prediction of infertility and early planning of an appropriate therapy. Methods: A clinical and molecular genetic investigation was performed in all members of a Chinese family with BPES. Genomic DNA was extracted, and the FOXL2 coding region was sequenced. Subcellular localization was performed by confocal microscopy. Transactivation studies were performed by real-time PCR, dual luciferase reporter assays and electrophoretic mobility shift assays. Results: A novel deletion mutation (C.634_641 del, CCCATGC) between the forkhead domain and the polyalanine domain was found, resulting in a frameshift mutation and a truncated protein. Functional studies showed a strong cytoplasmic mislocalization and abnormal transactivation activity, implying a type I kind mutation with a large chance of infertility. Conclusion: This study identifies that this mutation indicates the probability of developing into POF and shows the importance and necessity of early recognition of BPES type through mutation testing for female patients. Prompt personalized therapy and followup is of great clinical significance for female patients carrying this kind of mutation.
\end{abstract}




\section{Introduction}

Blepharophimosis-ptosis-epicanthus inversus syndrome (BPES; OMIM 110100) is a rare autosomal dominant genetic disorder with an estimated incidence of one in 50, 000[1]. It is characterized by narrow horizontal palpebral fissures, congenital ptosis, telecanthus and epicanthus inversus [2]. In addition to the ophthalmic symptoms, BPES patients may have female infertility caused by premature ovarian failure (POF), classified as type I BPES, and those without POF are classified as type II [3]. Currently, mutations of the FOXL2 gene are considered to be the major cause of BPES [4].

FOXL2 is a 2.7-kb gene with a single exon that encodes a 376 amino acid protein, which contains a 110 amino acid DNA-binding forkhead domain (FHD) and a polyalanine (poly-Ala) tract of 14 residues of unknown function. FOXL2 is an essential transcription factor involved in normal development of the ovary and eyelid $[5,6]$. FOXL2 has also been suggested to be involved in the regulation of cholesterol and steroid metabolism, apoptosis, reactive oxygen species detoxification and inflammatory processes [7]. FOXL2 mutations are also involved in other diseases. In an Indian family with BPES, a FOXL2 mutation was found to be associated with polycystic ovary syndrome (PCOS) [8]. A recent genome-wide association study of Japanese individuals showed a significant association of keloid with FOXL2 expression. It has been assumed that gonadal and steroid hormones might influence keloid formation, which is known to be regulated by expression of FOXL2[9]. Shah et al. identified a somatic point mutation (C134W) and considered this mutant FOXL2 a potential driver in the pathogenesis of adult-type ovarian granulosa-cell tumour (GCT) [10]. Recent studies demonstrated a correlation between lacrimal gland underdevelopment and FOXL2[11], especially for truncated proteins or mutations affecting the FHD.

Thus far, $70 \%$ of BPES cases were shown to have intragenic mutations, including premature stop codons, missense mutations, expansions of the region encoding the poly-Ala tract and frameshift mutations leading to a shorter or longer proteins [7], but the correlation between mutation types and BPES types still needs to be clarified. Poly-Ala expansion mutations often lead to BPES type II, and truncations before the poly-Ala tract always lead to BPES type I $[4,12]$. However, the effect of mutations containing an intact FHD and poly-Ala tract remains elusive $[13,14]$. More recently, a direct correlation between the transcriptional activity of FOXL2 variants, also known as risk of POF, and the BPES type was identified using luciferase-based promoter reporter assays [15]. These results further described the early detection of POF and emphasized the need for personalized therapy and follow-up in female patients carrying distinct FOXL2 mutations.

In our study, we identified a novel mutation in the FOXL2 gene from a Chinese family with BPES. A novel deletion mutation, which resulted in a truncated protein due to a frameshift causing a premature stop codon, was associated with BPES type I, indicating the need for follow-up in female patients with this type of mutation.

\section{Materials and Methods}

\section{Patients}

We identified a three-generation, 15-member Chinese family with multiple individuals suffering from BPES. This family consisted of a consanguineous two-generation BPES family in which two girls were simultaneously suffering from esotropia. We were unable to assess the BPES type for this family because the female patients were all prepubescent girls. Clinical examinations were carried out by an ophthalmologist, and the clinical features of the index patients are shown in Table 1. Photographs were taken before surgery for the assessment of BPES features. The proband (III:5), an 8-year-old girl, inherited the pathogenic gene from her father. All the patients showed typical features of BPES, including a small palpebral fissure, ptosis of the eyelids, epicanthus inversus, and telecanthus. Informed consent for research was obtained from all participants or their guardians according to the tenets of the Declaration of Helsinki and Guidance of Sample Collection of Human Genetic Diseases through the Ministry of Public Health of China. 


\section{Cellular Physiology Cell Physiol Biochem 2018;45:203-211 \begin{tabular}{l|l|l} 
and Biochemistry 10.1159/000486358 & $\begin{array}{l}\text { (c) } 2018 \text { The Author(s). Published by S. Karger AG, Basel } \\
\text { www.karger.com/cpb }\end{array}$
\end{tabular}}

Li et al.: a Novel Mutation Implying BPES Type I

Table 1. Clinical features of patients with BPES. ${ }^{1}$ Inner intercanthal distance (IICD), vertical interpalpebral fissure height (IPFH), horizontal palpebral fissure length (HPFL), right eye (RE), left eye (LE)

\begin{tabular}{|c|c|c|c|c|c|c|c|c|c|c|c|}
\hline \multirow[t]{3}{*}{ Patient } & \multicolumn{4}{|c|}{ Vision } & \multicolumn{2}{|c|}{ IPFH(mm) } & \multicolumn{2}{|c|}{ HPFL(mm) } & \multicolumn{2}{|c|}{ Levator function(mm) } & \multirow[t]{3}{*}{ Other anomalies } \\
\hline & \multirow[t]{2}{*}{ Age/sex } & & & \multirow[t]{2}{*}{$\operatorname{IICD}(\mathrm{mm})$} & & & & & & & \\
\hline & & $\mathrm{RE}$ & LE & & $\mathrm{RE}$ & LE & RE & LE & $\mathrm{RE}$ & LE & \\
\hline III:5 & $8 / \mathrm{F}$ & 0.6 & 0.5 & 38 & 2 & 2 & 20 & 20 & 1 & 1 & Esotropia \\
\hline III:6 & $6 / F$ & 0.5 & 0.5 & 40 & 1 & 1 & 19 & 19 & 1 & 1 & Esotropia \\
\hline III:7 & $4 / \mathrm{M}$ & Unco & rative & 42 & 1 & 1 & 19 & 19 & 1 & 1 & Uncooperative \\
\hline
\end{tabular}

\section{DNA extraction and sequencing}

Blood samples were collected from all the individuals in this family, and genomic DNA was extracted from the leukocytes of peripheral venous blood using Automatic Nucleic Acid Isolation System (QuickGene 800, Fujifilm Life Science, Tokyo, Japan). For mutation analysis, PCR amplification of the genomic fragments encompassing FOXL2 coding regions was performed using overlapping sets of primers [16]. Purified PCR products were sequenced in both directions on an ABI 3730 DNA sequencer (Applied Biosystems PerkinElmer) to confirm the mutations. The mutation was analysed using Finch TV software, and the sequences were compared with the reference from the GenBank database (NM_023067). The mutation nomenclature was based on the Human Genome Variation Society guidelines (http://varnomen.hgvs.org/ recommendations/general/). The possible pathogenicity of the mutation were predicted using SIFT [11] and MutationTaster [17].

\section{Plasmid construction}

Complementary DNA (cDNA) encoding the FOXL2 ORF was cloned and inserted into pLenti-CMV-EGFP3FLAG-PGK-Puro and pcDNA3.1, yielding EGFP-FOXL2-wild-type plasmid and pcDNA3.1-FOXL2-wild-type plasmid, respectively. The mutant expression vectors were obtained by PCR with wild-type plasmid as templates. Then, mutant plasmids were generated to obtain an EGFP-FOXL2-mutant plasmid and pcDNA3.1FOXL2-mutant plasmid. The reporter plasmid PGL3-StAR was constructed as described previously [18]. All constructs were sequenced to exclude additional mutations.

\section{Subcellular location}

COS-7 cells were cultured and grown to $40-50 \%$ confluence, and then, each well was transfected with EGFP-FOXL2-wild-type, EGFP-FOXL2-mutant, or EGFP-mutant small protein constructs separately. After 48 hours, confocal laser scanning microscopy was performed to confirm the localization of wild-type and mutant proteins.

\section{Real-time PCR}

MLTC-1 cells plated in 6-well plates were transfected with $4 \mu \mathrm{g}$ of pcDNA3.1-FOXL2-wild-type/mutant/ empty plasmid for 48 hours. Then, cells were harvested, and total mRNA was extracted from the cells using TRIzol (Invitrogen). Reverse transcription (RT)-PCR was performed with $2 \mu \mathrm{g}$ of RNA using Superscript II (Invitrogen). Quantitative real-time PCR was performed to identify endogenous StAR expression. The housekeeping gene GAPDH was used as an endogenous control.

\section{Luciferase reporter assays}

CHO cells plated in 24-well plates were transfected with PGL3-StAR $(0.5 \mu \mathrm{g} /$ well $)$ plus different doses of pcDNA3.1-FOXL2-wild-type/mutant plasmid, including 125, 250, and $400 \mathrm{ng}$. The empty pcDNA3.1 plasmid was added at a concentration of $1 \mu \mathrm{g} / \mathrm{cell}$. The PRL-TK vector served as an indicator to estimate the transfection efficiency by constitutively expressing Renilla luciferase. Luciferase assays were conducted 
using the Dual Luciferase Reporter Assay System (Promega). Transfected cells were lysed, and the supernatant was collected after a brief centrifugation. Luciferase activity was measured according to the manufacturer's instructions on a Lumimark luminometer (Bio-Rad Laboratories, Hercules, CA, USA).

\section{Electrophoretic mobility shift assay}

For preparation of fluorescent FAM-labelled probes, the promoter region of StAR-1 and StAR-2 was PCR-amplified with Dpx DNA polymerase (TOLO Biotech, Shanghai) using the primers described previously [24]. The FAM-labelled probes were purified using the Wizard $\AA$ SV Gel and PCR Clean-Up System (Promega) and quantified with a NanoDrop 2000C. EMSA was performed in a 20- $\mu$ reaction volume containing $40 \mathrm{ng}$ of probe and various cell lysates. After incubation for $30 \mathrm{~min}$ at $37^{\circ} \mathrm{C}$, the reaction system was loaded onto a $10 \%$ PAGE gel buffered with $0.5 \times$ TBE. Gels were scanned with ImageQuant LAS 4000 mini (GE Healthcare).

\section{Results}

\section{Clinical and genetic features of patients with a novel mutation}

We identified a three-generation, 15-member Chinese family with multiple individuals suffering from BPES, as shown in Fig. 1A. All patients in this family demonstrated the typical features of BPES, including small palpebral fissures, ptosis, telecanthus and epicanthus inversus. None of the patients exhibited microcephaly or intellectual disability (Fig. 1B). From the clinical data (Table 1), female patients could not determine whether they would have POF because hormone levels, and fertility could not be evaluated.

Table 2. FOXL2 mutation (c.634_640 del CCCATGC) results from pathogenicity prediction software

\begin{tabular}{lrr}
\hline Software & SIFT & Mutation Taster \\
\hline Prediction Result & Affect protein function & Disease causing \\
\hline
\end{tabular}

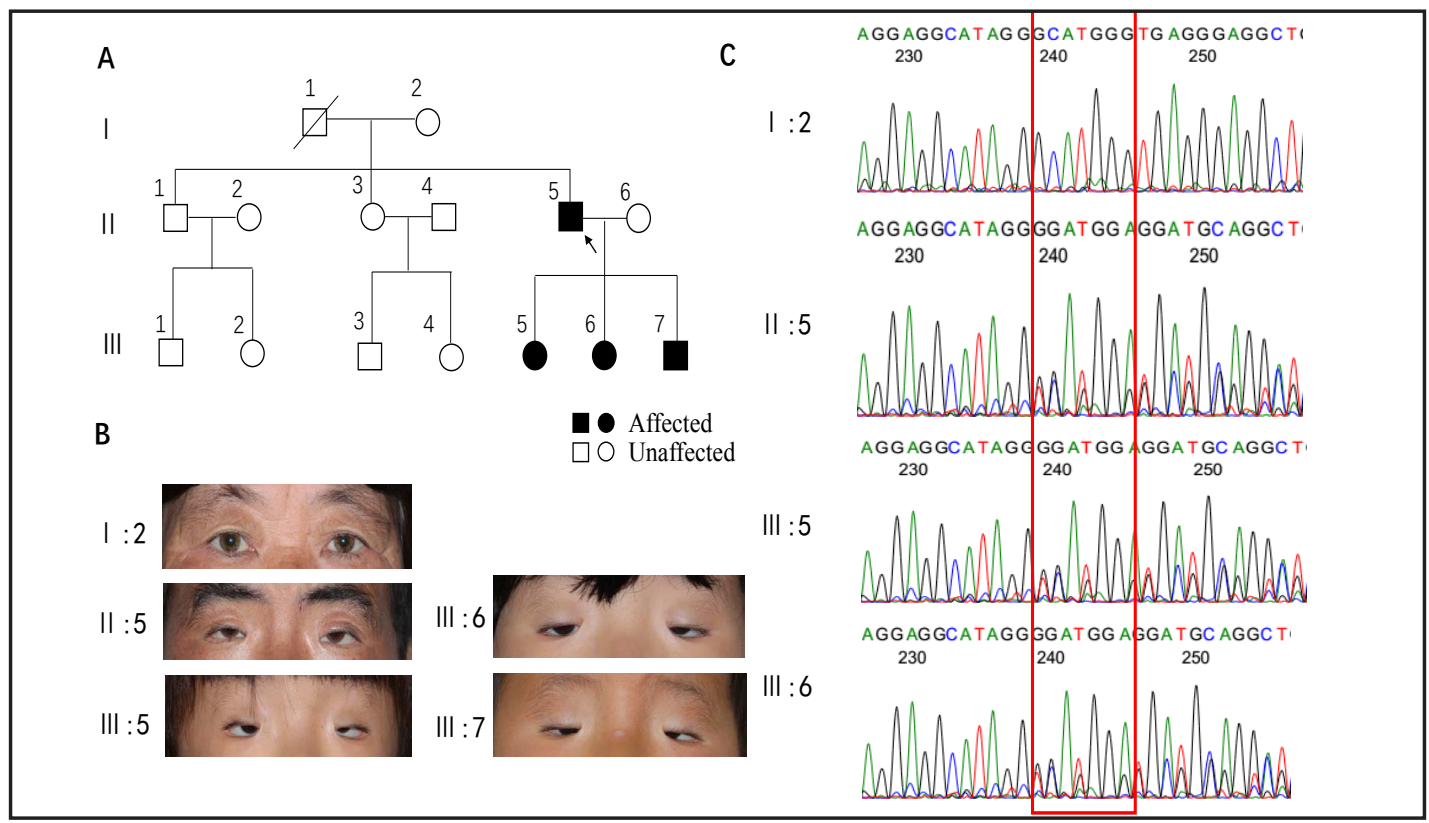

Fig. 1. Pedigrees. Facial photographs and genomic analysis of the FOXL2 gene. (A) Detailed pedigrees of the BPES cases included in the study. Affected individuals are indicated with closed symbols. The proband is indicated with an arrow. Roman numerals indicate the generation, and Arabic numerals indicate the individual number. (B) Facial photographs of the proband and the 4 members in this family. (C) Genomic sequencing analysis showed the 7-bp deletion c.634_641 del CCCATGC (Y215Pfs*54) in the FOXL2 gene, and the mutation was found in all affected members of this family. This variant was absent in 100 control individuals, including 1 relative of the affected families. 


\section{Cellular Physiology

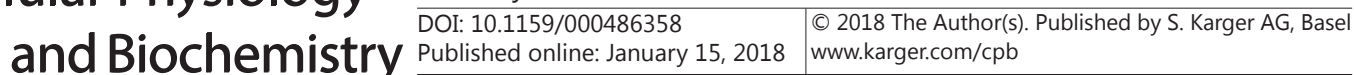

The sequence of the cloned PCR products obtained from these patients revealed a novel deletion mutation, c.634_641 del CCCATGC, a 7-bp deletion (Fig. 1C). This mutation is a p.Y215Pfs*54, resulting in a truncated protein due to a premature stop codon with a substitution of 54 amino acids, and the new small protein consists of 81 amino acids (Fig. 2).

This mutation change was predicted to be not tolerated by SIFT (http://sift.bii.a-star. edu.sg/index.html) and to be a disease-causing variant by Mutation Taster (http://www. mutationtaster.org/; Table 2). However, for the female patients in this family, whether this mutation results in female infertility (POF) remains to be determined.

Mutation impairs subcellular
localization
To determine the type of BPES, we first performed cellular location studies in COS7 cells. Three constructs containing a EGFP-FOXL2-WT fusion protein, EGFP-FOXL2-mutant fusion protein and EGFP-MT-small protein were transfected into COS-7 cells. As shown, cells transfected with the mutant protein showed a cytoplasmic localization, whereas wild-type proteins showed a location of nucleus (Fig. 3).

Functional assays of mutation on transcriptional activity of FOXL2

Next, we tested whether mutant proteins have transactivation capacity. First, we measured the expression of the FOXL2 target genes StAR, SIRT1 and OSR2 in MLTC-1 cells that were transfected with wild-type or mutant FOXL2. Real-time PCR assays showed that expression of the target genes displayed no significant changes when transfected with the mutant protein, whereas wild-type FOXL2 significantly decreased the StAR level and increased expression of OSR2 and SIRT1 (Fig. 4A).

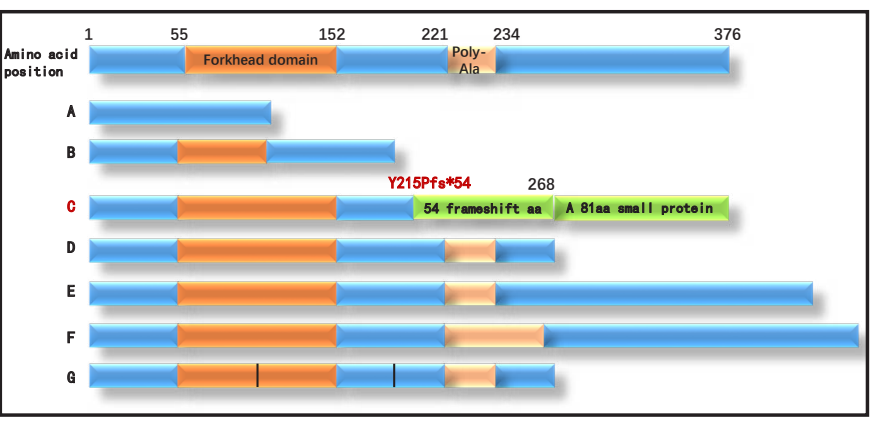

Fig. 2. Mutations were classified into 7 groups according to De Baere et al.[13]. A: A truncated protein without an FHD; B: A truncated protein with a partial FHD; C: Nonsense mutations leading to predicted truncated proteins with a complete FHD and without the poly-Ala tract. D: Frameshift mutations leading to a truncated protein with a complete FHD and poly-Ala domain. E: Frameshift mutations leading to an elongated FOXL2 protein with a complete FHD and poly-Ala domain; F: In-frame mutation; G: Missense mutation. This novel mutation belongs to type $\mathrm{C}$, a truncated protein that consists of 268 amino acids, and an 81-aa small protein.

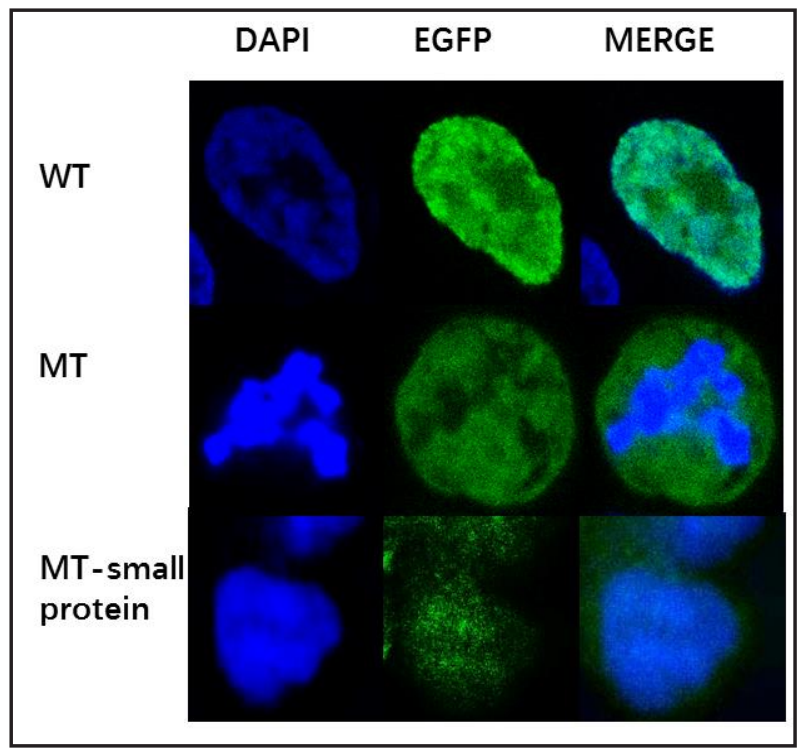

Fig. 3. Subcellular localization of wild-type, mutant FOXL2, and mutant small protein. The left panel shows nuclear staining with DAPI. The middle panel is a representative image showing the subcellular localization of FOXL2 as a fusion protein with EGFP. The right panel is a merged image of the previous two images. In contrast to the wild-type FOXL2, the mutant protein was retained in the cytoplasm, and the small protein was located in both the nucleus and cytoplasm. 
Fig. 4. Functional assay of FOXL2 transactivation capacity. (A) Relative mRNA expression of StAR, SIRT1 and OSR2 in cells transiently transfected with pcDNA3.1 vector, wild-type FOXL2 and mutant FOXL2. (B) Reporter gene assay showing that mutant FOXL2 cannot repress StAR expression in a dose-dependent manner similar to wildtype. (C) EMSA indicates that the wild-type FOXL2 protein binds to the StAR promoter (lane 3), but the mutant protein abrogates these interactions (lane 4). A 100-fold unlabelled probe was used to demonstrate specific binding between FOXL2 and the StAR promoter (lane5). The FOXL2-StAR promoter complexes are indicated with rectangles.

Then, reporter assays were performed. CHO cells were co-transfected with wild-type or mutant FOXL2 plasmid and the reporter construct PGL3-STAR containing the StAR promoter. As expected, the wild-type FOXL2 construct suppressed the activity of the StAR promoter (Fig. 4B), while mutant protein showed decreased repression of StAR.

Further, EMSAs were performed to test the binding of the FOXL2 protein and StAR promoter. As shown, wild-type FOXL2 protein binds to StAR probes, but the mutant lacked this binding ability (Fig. 4C). Then, 100-fold unlabelled probe was added, and the binding was partly inhibited.

\section{Discussion}

In this study, we reported a novel deletion mutation between the FHD and polyAla domain of the FOXL2 gene, which resulted in a truncated protein due to a frameshift leading to a premature stop codon. According to the mutation prediction software SIFT and Mutation Taster, the deletion may affect the protein function and may be a disease-causing variant. Since this family only includes one adult male patient and two girl patients, the type of BPES could not be identified clinically. Thus, we could not determine whether the two girl patients will develop POF in the future.

To identify the type of novel mutation in this family and to provide the patients with advice on whether they should focus on ovarian development and function, we examined the effect of the FOXL2 mutant.

First, we tested the subcellular localization of the truncated mutant protein. The results showed cytoplasmic mislocalization of the truncated protein and no difference in the distribution of the abnormally small mutant protein. Since poly-Ala-containing proteins tend to concentrate in nuclear subcompartments as transcription factors [12, 19], it is not 


\section{Cellular Physiology Cell Physiol Biochem 2018;45:203-211

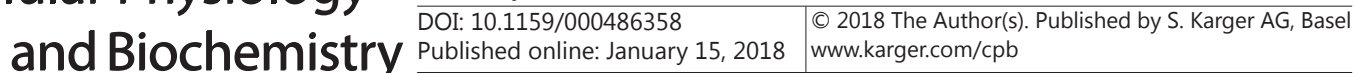 \\ Li et al.: a Novel Mutation Implying BPES Type I}

surprising that the mutant protein showed cytoplasmic retention in the absence of poly-Ala. However, misfolding of FOXL2 might bury the nuclear localization signal (NLS) and interact with cytoplasmic proteins that retain FOXL2[20]. Cytoplasmic retention is a weak predictor of BPES types because p.I80T and p.I84S lead to type I BPES, inducing cytoplasmic retention, while p.Y215C, which results in type BPES II, also causes a similar phenotype [15].

Thus, molecular behaviours were examined to distinguish between these two types. Real-time PCR assays showed that wild-type FOXL2 can repress the expression of StAR, a marker of granulosa cell differentiation, upregulate the expression of SIRT1, a deacetylase, which in turn decreases the transactivation ability of FOXL2, and a crucial FOXL2 ovarian target $[15,18]$, and increase the expression of OSR2, which is important for craniofacial development [12]. However, these characteristics were not observed with mutant FOXL2, suggesting that a functional loss mutation affects both eyelid and ovarian development.

Among the target genes, StAR plays a crucial role in early functional maturation of ovarian antral follicles when FOXL2 expression is decreased [19]. Thus, mutant FOXL2 would result in accelerated differentiation of granulosa cells, ultimately leading to POF [15]. Therefore, target gene promoter activity assay was performed to confirm that reduced transcriptional repression of the StAR promoter was associated with the mutant FOXL2 protein. Finally, EMSAs further confirmed the loss of repressor activity of wild-type FOXL2 by reducing binding of the FOXL2 protein to the StAR promoter. All these results indicated that this novel deletion mutation may be associated with BPES type I.

The distinction between these two types is critical for female patients, as it may allow for prediction of fertility and appropriate therapy [21]. According to De Baere [5, 22], mutations are classified into 7 groups according to their effect on the predicted protein (Fig. 2). Our novel mutation belongs to group $C$, which usually results in BPES type I, consistent with our results. The novel C.634_641 del, CCCATGC mutation was located in the coding region between the FHD and poly-Ala tract, which has rarely been reported. Other mutations identified in the same coding region include c.627delT (type I) [23], c.475dupC (type II) [24], c.644A>G (type II), c.536C > G (type II) [20], and c.650C > G (type II)[25]. Point mutations may not be related to POF, but truncated proteins are more likely to be correlated with POF. However, more studies are required to identify the relationships between mutation type and their location. Furthermore, functional assays are needed for confirmation of the effects of the mutations.

Taken together, our results provide functional evidence in favour of BPES type I. Our study aims to highlight the importance of early recognition of mutation type and emphasize the need for personalized therapy and follow-up in female patients carrying this type of mutation.

\section{Acknowledgements}

We are very grateful to the family and the volunteers who participated in this study and to the clinicians and researchers who made this work possible.

This work was supported by the National Natural Science Foundation of China (Grant 31470757, 81402258), the ShuGuang Project of Shanghai Municipal Education Commission and Shanghai Education Development Foundation (17SG19), the Outstanding Yong Medical Scholar of Shanghai Municipal Commission of Health and Family Planning(2017YQ067), the Program for Professor of Special Appointment (Eastern Scholar) at the Shanghai Institutions of Higher Learning (1410000159), the SMC-ChenXing Yong Scholar Program (2014, Class B; 2016,Clss A), the Shanghai Municipal Education Commission-Gaofeng Clinical Medicine Grant (20161317), the Outstanding Yong Scholar Grant of Shanghai JiaoTong University School of Medicine (16XJ11002), the Research physician of Shanghai JiaoTong University School of Medicine, the Science and Technology Commission of Shanghai (16ZR1419600, 17DZ2260100). 


\section{Cellular Physiology Cell Physiol Biochem 2018;45:203-211

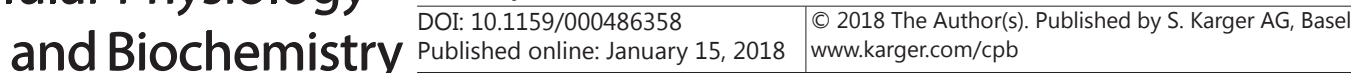

Li et al.: a Novel Mutation Implying BPES Type I

Disclosure Statement

The authors declare that they have no Disclosure Statement.

\section{References}

$>1$ Oley C, Baraitser M: Blepharophimosis, ptosis, epicanthus inversus syndrome (BPES syndrome). J Med Genet 1988;25:47-51.

$\longrightarrow 2$ Beaconsfield M, Walker JW, Collin JR: Visual development in the blepharophimosis syndrome. Br J Ophthalmol 1991;75:746-748.

-3 Zlotogora J, Sagi M, Cohen T: The blepharophimosis, ptosis, and epicanthus inversus syndrome: delineation of two types. Am J Hum Genet 1983;35:1020-1027.

4 Crisponi L, Deiana M, Loi A, Chiappe F, Uda M, Amati P, Bisceglia L, Zelante L, Nagaraja R, Porcu S, Ristaldi MS, Marzella R, Rocchi M, Nicolino M, Lienhardt-Roussie A, Nivelon A, Verloes A, Schlessinger D, Gasparini P, Bonneau D, Cao A, Pilia G: The putative forkhead transcription factor FOXL2 is mutated in blepharophimosis/ptosis/epicanthus inversus syndrome. Nat Genet 2001;27:159-166.

5 Beysen D, De Paepe A, De Baere E: FOXL2 mutations and genomic rearrangements in BPES. Hum Mutat 2009;30:158-169.

6 Cocquet J, Pailhoux E, Jaubert F, Servel N, Xia X, Pannetier M, De Baere E, Messiaen L, Cotinot C, Fellous M, Veitia RA: Evolution and expression of FOXL2. J Med Genet 2002;39:916-921.

7 Moumne L, Batista F, Benayoun BA, Nallathambi J, Fellous M, Sundaresan P, Veitia RA: The mutations and potential targets of the forkhead transcription factor FOXL2. Mol Cell Endocrinol 2008;282:2-11.

8 Kaur I, Hussain A, Naik MN, Murthy R, Honavar SG: Mutation spectrum of fork-head transcriptional factor gene (FOXL2) in Indian Blepharophimosis Ptosis Epicanthus Inversus Syndrome (BPES) patients. Br J Ophthalmol 2011;95:881-886.

-9 Nakashima M, Chung S, Takahashi A, Kamatani N, Kawaguchi T, Tsunoda T, Hosono N, Kubo M, Nakamura Y, Zembutsu H: A genome-wide association study identifies four susceptibility loci for keloid in the Japanese population. Nat Genet 2010;42:768-771.

10 Shah SP, Kobel M, Senz J, Morin RD, Clarke BA, Wiegand KC, Leung G, Zayed A, Mehl E, Kalloger SE, Sun M, Giuliany R, Yorida E, Jones S, Varhol R, Swenerton KD, Miller D, Clement PB, Crane C, Madore J, Provencher D, Leung P, DeFazio A, Khattra J, Turashvili G, Zhao Y, Zeng T, Glover JN, Vanderhyden B, Zhao C, Parkinson CA, Jimenez-Linan M, Bowtell DD, Mes-Masson AM, Brenton JD, Aparicio SA, Boyd N, Hirst M, Gilks CB, Marra M, Huntsman DG: Mutation of FOXL2 in granulosa-cell tumors of the ovary. N Engl J Med 2009;360:2719-2729.

11 Kumar P, Henikoff S, Ng PC: Predicting the effects of coding non-synonymous variants on protein function using the SIFT algorithm. Nat Protoc 2009;4:1073-1081.

12 Caburet S, Demarez A, Moumne L, Fellous M, De Baere E, Veitia RA: A recurrent polyalanine expansion in the transcription factor FOXL2 induces extensive nuclear and cytoplasmic protein aggregation. J Med Genet 2004;41:932-936.

13 De Baere E, Beysen D, Oley C, Lorenz B, Cocquet J, De Sutter P, Devriendt K, Dixon M, Fellous M, Fryns JP, Garza A, Jonsrud C, Koivisto PA, Krause A, Leroy BP, Meire F, Plomp A, Van Maldergem L, De Paepe A, Veitia R, Messiaen L: FOXL2 and BPES: mutational hotspots, phenotypic variability, and revision of the genotypephenotype correlation. Am J Hum Genet 2003;72:478-487.

14 De Baere E, Copelli S, Caburet S, Laissue P, Beysen D, Christin-Maitre S, Bouchard P, Veitia R, Fellous M: Premature ovarian failure and forkhead transcription factor FOXL2: blepharophimosis-ptosis-epicanthus inversus syndrome and ovarian dysfunction. Pediatr Endocrinol Rev 2005;2:653-660.

15 Dipietromaria A, Benayoun BA, Todeschini AL, Rivals I, Bazin C, Veitia RA: Towards a functional classification of pathogenic FOXL2 mutations using transactivation reporter systems. Hum Mol Genet 2009;18:3324-3333.

16 Fan JY, Han B, Qiao J, Liu BL, Ji YR, Ge SF, Song HD, Fan XQ: Functional study on a novel missense mutation of the transcription factor FOXL2 causes blepharophimosis-ptosis-epicanthus inversus syndrome (BPES). Mutagenesis 2011;26:283-289.

17 Schwarz JM, Rodelsperger C, Schuelke M, Seelow D: MutationTaster evaluates disease-causing potential of 


\section{Cellular Physiology Cell Physiol Biochem 2018;45:203-211

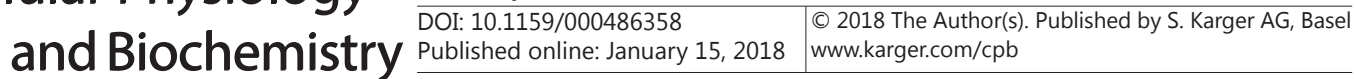

Li et al.: a Novel Mutation Implying BPES Type I

sequence alterations. Nat Methods 2010;7:575-576.

18 Fan J, Zhou Y, Huang X, Zhang L, Yao Y, Song X, Chen J, Hu J, Ge S, Song H, Fan X: The combination of polyalanine expansion mutation and a novel missense substitution in transcription factor FOXL2 leads to different ovarian phenotypes in blepharophimosis-ptosis-epicanthus inversus syndrome (BPES) patients. Hum Reprod 2012;27:3347-3357.

19 Caburet S, Vaiman D, Veitia RA: A genomic basis for the evolution of vertebrate transcription factors containing amino Acid runs. Genetics 2004;167:1813-1820.

20 Nallathambi J, Laissue P, Batista F, Benayoun BA, Lesaffre C, Moumne L, Pandaranayaka PE, Usha K, Krishnaswamy S, Sundaresan P, Veitia RA: Differential functional effects of novel mutations of the transcription factor FOXL2 in BPES patients. Hum Mutat 2008;29:E123-131.

-21 Nuovo S, Passeri M, Di Benedetto E, Calanchini M, Meldolesi I, Di Giacomo MC, Petruzzi D, Piemontese MR, Zelante L, Sangiuolo F, Novelli G, Fabbri A, Brancati F: Characterization of endocrine features and genotypephenotypes correlations in blepharophimosis-ptosis-epicanthus inversus syndrome type 1. J Endocrinol Invest 2016;39:227-233.

-22 Cocquet J, De Baere E, Gareil M, Pannetier M, Xia X, Fellous M, Veitia RA: Structure, evolution and expression of the FOXL2 transcription unit. Cytogenet Genome Res 2003;101:206-211.

23 Correa FJ, Tavares AB, Pereira RW, Abrao MS: A new FOXL2 gene mutation in a woman with premature ovarian failure and sporadic blepharophimosis-ptosis-epicanthus inversus syndrome. Fertil Steril 2010;93:1006 e1003-1006.

24 Jiang H, Huang X, Su Z, Rao L, Wu S, Zhang T, Li K, Quan Q, Zhang K: Genetic analysis of the forkhead transcriptional factor 2 gene in three Chinese families with blepharophimosis syndrome. Mol Vis 2013;19:418-423.

-25 Haghighi A, Verdin H, Haghighi-Kakhki H, Piri N, Gohari NS, De Baere E: Missense mutation outside the forkhead domain of FOXL2 causes a severe form of BPES type II. Mol Vis 2012;18:211-218. 